\title{
Belphégor
}

Curreri, Luciano et Michel Delville (s. la dir. de). Il grande "incubo che mi son scelto" - Le grand "cauchemar de mon choix" - The Big "Nightmare of my choice". Prove di avvicinamento a Profondo rosso / Les frissons de l'angoisse / Deep Red (1975-2015)

\section{Vittorio Frigerio}

\section{(2) OpenEdition Journals}

\section{Electronic version}

URL: http://journals.openedition.org/belphegor/717

DOI: 10.4000/belphegor.717

ISSN: 1499-7185

Publisher

LPCM

\section{Electronic reference}

Vittorio Frigerio, "Curreri, Luciano et Michel Delville (s. la dir. de). II grande "incubo che mi son scelto" - Le grand "cauchemar de mon choix" - The Big "Nightmare of my choice". Prove di avvicinamento a Profondo rosso / Les frissons de l'angoisse / Deep Red (1975-2015) », Belphégor [Online], 14 | 2016, Online since 16 July 2016, connection on 24 September 2020. URL : http://journals.openedition.org/ belphegor/717 ; DOI : https://doi.org/10.4000/belphegor.717

This text was automatically generated on 24 September 2020.

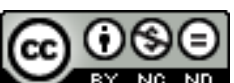

Belphégor est mis à disposition selon les termes de la Licence Creative Commons Attribution - Pas d'Utilisation Commerciale - Pas de Modification 4.0 International. 


\section{Curreri, Luciano et Michel Delville (s. la dir. de). Il grande "incubo che mi son scelto" - Le grand "cauchemar de mon choix" - The Big "Nightmare of my choice". Prove di avvicinamento a Profondo rosso / Les frissons de l'angoisse / Deep Red (1975-2015)}

Vittorio Frigerio

\section{REFERENCES}

Curreri, Luciano et Michel Delville (s. la dir. de). Il grande "incubo che mi son scelto" Le grand "cauchemar de mon choix" - The Big "Nightmare of my choice". Prove di avvicinamento a Profondo rosso / Les frissons de l'angoisse / Deep Red (1975-2015). Piombino: Edizioni Il Foglio, 2015. 135 p. ISBN: 978-88-7606-597-2. 


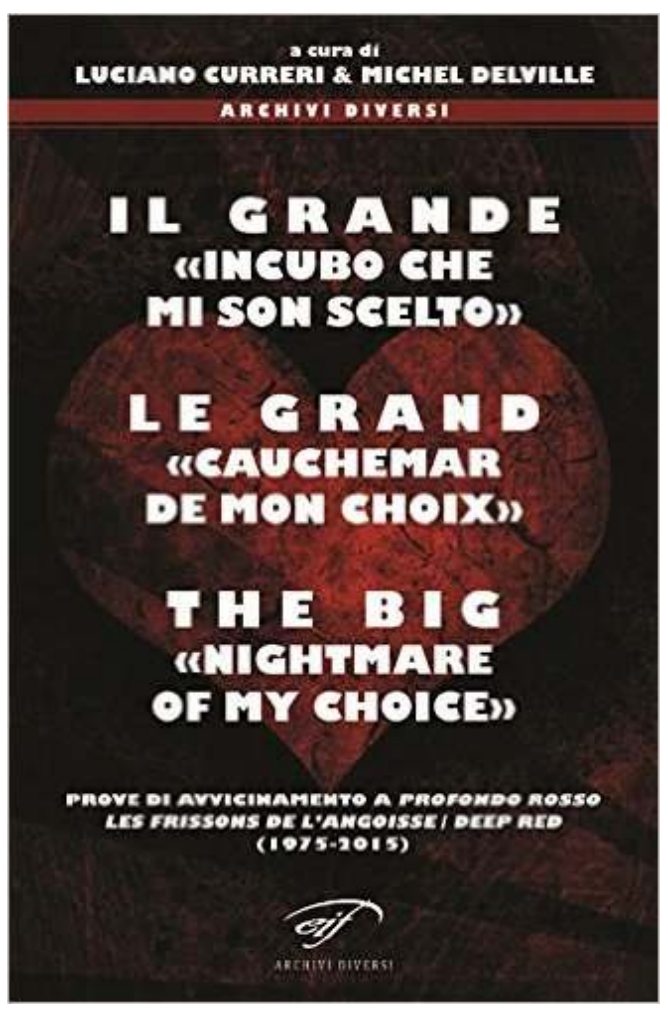

1 Ce volume, petit mais dense, soumet au regard croisé de douze critiques un des films cult les plus appréciés du célèbre cinéaste italien Dario Argento, pour fêter le quarantième anniversaire de sa sortie et évaluer l'influence qu'il a pu avoir sur la culture, au sens large, de notre époque. Les lecteurs de Belphégor reconnaîtront sans peine dans l'attitude décontractée et sans prétentions, tout en restant rigoureuse, et dans l'importance accordée au multilinguisme (l'introduction et trois articles sont en italien, quatre en français et quatre autres en anglais), l'approche qu'a toujours privilégiée notre revue dans l'analyse de ces objets culturels multiples et difficiles à classer, appartenant au domaine protéiforme de la culture de masse.

Dans son introduction, dans une défense passionnée de la valeur de l'ouverture à d'autres idiomes et d'autres traditions épistémologiques, Luciano Curreri parle du film comme d'une expérience d'ordre dysesthésique, qui est près d'atteindre au « sublime ». Il y a un terme qui revient à plusieurs reprises et de différentes manières, et qui résume en définitive l'attitude mentale des directeurs du volume : "sporco", "sale », dans le sens d'énergique, peu soucieux de règles étouffantes et bien peu souvent aussi utiles qu'elles prétendent l'être, disposé au métissage et à la contamination.

Dans le premier article («Figli deformi e prole maledetta : l'influenza di Profondo rosso nella letteratura horror italiana degli anni Novanta »), Daniele Comberati se penche sur l'influence des travaux d'Argento sur la production de films d'horreur des années quatre-vingt-dix, période à laquelle il attribue une valeur de charnière entre le développement du genre et son acceptation ultime dans le panorama culturel contemporain. Le critique retrace les signes laissés par le film dans trois ouvrages de cette période : le recueil de nouvelles Sogni di sangue de Tiziano Sclavi (le premier scénariste de la bande dessinée Dylan Dog, publiée par la maison d'édition Bonelli, qui a fait beaucoup pour permettre le passage de l'horreur des marges de la littérature jusqu'en son cœur), Le radici del male, d'Ada Teodorami, représentante du groupe 
d'écrivains connus sous le nom de "cannibales », et Faccia di sale, d'Eraldo Baldini. S'agit-il d'un choix arbitraire? C'est le critique lui-même qui le suggère, tout en relevant l'omniprésence de l'esthétique typique de Dario Argento dans la production «horror» de la période, telle qu'elle justifierait des comparaisons avec pratiquement n'importe quel ouvrage appartenant à ce genre paru pendant ces années, où l'influence du cinéaste s'élargissait comme une tache d'huile - ou de sang.

Dans un article riche en références, allusions et renvois " Tutto per un articolo di merda ?' Giornalisti tra artisti e assassini in Il gatto a nove code e Profondo rosso (non senza qualche riferimento a L'uccello dalle piume di cristallo e Quattro mosche di velluto grigio) » Luciano Curreri traite du personnage du journaliste et disserte librement des ressemblances diverses et variées entre artistes et assassins, au sein d'une « cinématographie dans laquelle la prévalence du visuel par rapport à l'intrigue » est une constante essentielle. Après lui, Mauro Giori («Gli ‘strani gusti sessuali’ di Carlo. Profondo rosso come psicopatologia hitchcockiana 'tollerante'») se penche sur les personnages homosexuels apparaissant dans les films d'Argento, à la lumière de l'influence d'Hitchcock et de la théorie psychanalytique. Il retrouve dans le cinéaste italien (qui justifiait pour des raisons de «réalisme » et d'expérience personnelle la présence de personnages gay dans ses films) « un mélange de pulsions variées », dont le résultat finit par être caractérisé par une «forme particulièrement autochtone (nostrana) de tolérance ".

5 Michel Delville ( On the Giallo considered as one of the Fine Arts (with Attendant Considerations of the Weird Tale, the Dark Grotesque and Argento's Philosophy of Composition) ») retrouve chez Argento un fond qu'il aurait en commun avec Quincey, à travers une esthétisation du meurtre où la dimension éthique, quoique non entièrement absente, passe au second plan. Au-delà des inévitables références à Hitchcock, il met à contribution également Edgar Allan Poe, dans le but d'éclairer la «moral polymorphousness » du film, qui ne saurait supporter des frontières par trop clairement délimitées. Lovecraft trouve aussi sa place dans l'analyse, vu comme une clef utile pour comprendre ce que le critique appelle « the complex and intense matrix of associational interrelations and creative tensions » qui se trouve à la base de l'œuvre du cinéaste.

6 Mark Duffet («Casting call : Profondo rosso and Blow-up») approche le travail de Dario Argento à la lumière de celui d'Antonioni. Mais pour y arriver, il fait également un détour important - ainsi que les critiques précédents - du côté de Psycho. C'est à travers l'identification de certaines «anomalies » dans l'intrigue que sont ainsi proposés des parcours intertextuels qui font du film d'Antonioni une clef de lecture possible pour celui d'Argento, et cela à travers la figure de l'acteur David Hemmings, qui joue dans les deux films.

7 Alexandra Heller-Nicholas et Craig Martin («Beyond the Evil Child: Knowledge and Age in Profondo rosso ») explorent la représentation de l'enfance, de la victime au monstre assassin, mais aussi la possibilité de l'existence d'un autre regard, susceptible de déstabiliser celui, autoritaire, de l'adulte et de suggérer l'impossibilité de la formulation de jugements éthiques inchangeables.

8 Peter Hutchings («Deep Red: The Art of Violence ») offre une lecture du film vu comme une synthèse ultime de formes et expressions culturelles variées, du théâtre à la littérature, à la peinture, à l'architecture, transformées en véhicules cinématographiques de la folie et de la violence. À travers de nombreux exemples, le 
critique montre comment on peut retrouver dans ce film un «obsessive network of references to the connectedness of art with mental disturbance, violence and murder ».

Léopold Dubois («De Profundis») se concentre également sur le formalisme typique de l'œuvre d'Argento (il n'y a pratiquement pas d'article qui ne mentionne l'hommage présent dans le film au célèbre tableau de Hopper, Nighthawks) pour parvenir à la conclusion que Les frissons de l'angoisse est une "summa» de tous les éléments fondamentaux du giallo. Son analyse, solidement contextualisée dans le cadre de la production cinématographique du temps, s'intéresse avec acuité au rôle de la musique, utilisée "à contresens", avec une finalité esthétique que les critiques de l'œuvre auraient jusqu'ici peiné à reconnaître pleinement.

Jeremy Hamers (« Revoir Profondo rosso. Notes sur le retard d'une distinction sociale.») choisit quant à lui d'aller outre le réseau complexe des allusions et de l'intertextualité, pour découvrir dans le film ce qui est généralement considéré absent presque par définition dans le giallo de l'époque : le discours social. Discours qu'il considère bien au contraire comme étant au cœur même de l'œuvre, tellement évident que, paradoxalement, il ne peut pour finir qu'être ignoré.

11 Dick Tomasovic ("Contre le sujet. Profondo rosso ou le spectateur inquiété ») consacre son étude à une lecture attentive et détaillée des images, mettant en évidence le «byzantinisme» d'Argento et l'ambiguïté fondamentale de ses représentations, qui fonctionnent comme un authentique carrousel de points de vue détaché de toute règle narrative, permettant un jeu au plaisir toujours renouvelé dans les méandres de l'esthétique, au milieu d'allusions et de renvois tout aussi riches que contradictoires et déstabilisants.

12 En dernier, Laurent Vauclaire («Espace du discours et discours de l'espace: la picturalité à l'épreuve du mouvement ») montre comment l'usage de l'espace de la part des personnages, ainsi que la compréhension qu'ils en ont, détermine leur destin. Lire l'espace signifie alors le dominer, pour l'assassin aussi bien que pour le héros et, au bout du compte, pour le spectateur.

13 Ce qu'il ne faudra pas venir chercher dans ce volume est une véritable introduction à Argento et à son œuvre, susceptible de permettre au lecteur qui ne serait pas déjà familier du sujet de s'y retrouver. Mais la «sale douzaine » de spécialistes dont les écrits - intéressants et complémentaires - se retrouvent ici auront réussi avec élégance à atteindre leur but : montrer comment Profondo rosso a pu développer un vaste réseau de racines dans le sous-sol de la culture italienne (et pas exclusivement dans les chasses gardées du genre « horror »), des rhizomes à la Deleuze susceptibles de laisser émerger à l'imprévu les pousses les plus surprenantes.

\section{AUTHOR}

\section{VITTORIO FRIGERIO}

Dalhousie University 